\title{
Preparation and cytotoxic activity of resveratrol-gold nanoparticles conjugated to folic acid against MCF-7 cell line
}

\author{
Muhammad SULAIMAN 1 (D), Sutriyo SUTRIYO ${ }^{1 *}$ (D), Abdul MUN'IM ${ }^{1}$ \\ 1 Faculty of Pharmacy, University of Indonesia, Depok, Indonesia. \\ * Corresponding Author. E-mail: sutriyo@farmasi.ui.ac.id (S.S.); Tel. +62-21-7864049.
}

Received: 30 November 2018 / Revised: 10 March 2019 / Accepted: 14 March 2019

\begin{abstract}
The potential benefits of resveratrol (Trans-3, 5, 4'-trihidroxy stilbene) as an anti-cancer are lowered because of it's low aquous solubility, instability and low bioavailability. Recent progresses in nanoparticle technology offer a promising loophole to solve many problems in developing theurapetic agents. The ability of a nanocarrier to selectively bring drug substances to the site of a tumor solely based on passive targeting can be enhanced by coupling a ligand to the surface of the nanocarrier knowingly as active targeting. This research aimed to test the cytotoxic activity of resveratrol conjugated to gold nanoparticles-folate as a carrier. Gold nanoparticles (AuNp) was chosen as a suitable carriers to increase the potency of resveratrol (Rsv) as an anti cancer agent with the help of folic acid (FA). The expression of folate receptors are hightened due to the excessive need of folic acid by certain types of cancer cells. Thus folic acid can act as a targeting agent to help introduce gold nanoparticles to the target cells. Furthermore, the cytotoxic activity of the final conjugate (FA-AuNp-Rsv) will be tested against human breast cancer MCF-7 cell line using MTT cholorimetric test. Gold nanoparticles were fabricated using optimized amount of polyvinyl alcohol (PVA) as a stabilizers. FA were attached to the surface of gold nanoparticles using 4-Aminothiophenol (4-Atp) as a linker. The carboxilic group of FA was first activated using common EDC-NHS reaction and reacted with amine group of 4-Atp to form amide bond. The physical and chemical characterization has been conducted and it was found that the final 86.9 nm nanoconjugate (FA-AuNp-Rsv) were successfuly synthesized with good stability and possesed an elevated activity $\left(\mathrm{IC}_{50}=21.28 \pm 1.04\right.$ $\mu \mathrm{M})$ compared to resveratrol alone $(49.94 \pm 1.06 \mu \mathrm{M})$. Thus this results were hoped to be an adequate addition to the development of nanotechnology in cancer therapy.
\end{abstract}

KEYWORDS: Cytotoxic activity; gold nanoparticles; resveratrol; MTT method; MCF-7 cells.

\section{INTRODUCTION}

Breast cancer is the second in terms of the highest cause of deaths which is responsible for about 198,000 deaths a year in a developed country or about $15.4 \%$ deaths around the world. In the United States, $12 \%$ or 1 out of 8 women suffers from breast cancer [1]. These facts motivate researches around the world to discover not only pharmacologically active substances but also the right technology to obtain an advanced and better treatment for breast cancer.

Beside the use of synthetic drugs which are mostly happen to possess many side effects especially to the normal tissue, natural substances also have been found out to be beneficial and considered save. Resveratrol or trans-3,5,4'trihidroxy stilbene, a polyphenol derived from stilbens has been proved over decades to have many potentials such as anti-oxidants, anticarcinogenic, antiobesity and also protection against heart and brain diseases [2]. An earlier report proposed that caspase activation and apoptotic induction are resveratrol's main mechanism that responsible for inhibiting the progression of cancer cells [3]. Despite of its many benefits, many evidence have also risen regarding it's instability, poor solubility and low bioavailability as a result of rapid metabolism [4]. Nanoparticle based targeting system usually chosen to overcome these problems.

Systemic delivery of anticancer substances as a conventional therapy aimed to increase the amount of therapeutics substance into the site of action which nanocarrier system usualy served in this area through its ability to accumulate at tumor site. This term is known widely as enhanced permeability and retention (EPR). Gold nanoparticles (AuNp) is famous as a drug carrier for many cancer drugs through it's fine nature such as

How to cite this article: Sulaiman M, Sutriyo S, Mun'im A. Preparation and cytotoxic activity of resveratrol-gold nanoparticles conjugated to folic acid against MCF-7 cell line. J Res Pharm. 2019; 23(5): 927-934. 
non-toxic, inert and easily accumulate in the tumor site. Its tunable properties which is usually correspond with the physical attributes can be achieved through different synthesis methods [5]. Gold nanoparticles could be prepared by several different methods. These methods commonly divided in to two categories, the topdown method and bottom's up method. Upon the fabrication of gold nanoparticles, a stabilizing agent sometimes needed to prevent the particles to aggregate and form a larger particles. One of the most popular chemical method is the one that introduced by Brust et al where gold salts reduced by $\mathrm{NaBH}_{4}$ to form gold nanoparticles around 2-5 nm [6]. Many drugs and substances can also easily attached to the surface of gold nanoparticles (mostly through amine and thiol groups)[7].

A delivery and targeting system solely based on EPR effect simply can not guarantee that maximum amount of drugs can reach its pharmacological target. Without the help of a specific affinity, nanocarriers would risk to be redistributed in to vascular tissue [8]. While unspecific distribution of drugs can lead to increase of toxicity to the normal cells, ligands such as antibody or fragment, peptide and vitamins could work as targeting agents to help introduce drug substance into specific target location [9]. Folic acid receptors expression are high in over up to $40 \%$ of cancer cells type including breast cancer while that of normal cells are considered low [10]. This situation is due to the excessive need for folic acid by these type of cancer. This research aimed to elevate the potency of resveratrol as an anti cancer agent. In this research folic acid (FA) were attached to the surface of gold nanoparticles (AuNp) bearing resveratrol (Rsv) as the active agent. To examine the cytotoxic activity, we have tested the conjugate result (FA-AuNp-Rsv) against breast cancer MCF7 cells and compared the result to free reveratrol (Rsv). This research was only comparing the potency of resveratrol in conjugated form and the free form in a certain range of concentration. In this case, the cytotoxic activity of bare vehicle (AuNp) whether exist or not was not our strong discussion. FA was attached via amide bond link with 4 Aminothiophenol (4Atp) which acted as linker because of it's good thiols (S-H) affinity with AuNp suface. To our knowledge, this was the first attempt to conjugate resveratrol with gold nanoparticles bearing folate residues and see whether there was an elevated potency. As a complement in this research, in the beginning of the step, effect of different concentration of stabilizers were also be studied to optimize the stabilization of AuNp formation.

\section{RESULTS AND DISCUSSION}

\subsection{Characterization}

\subsubsection{Visual observation and $U V$-vis spectroscopic study}

Gold nanoparticles (AuNp) formation was fabricated by reducing $\mathrm{HAuCl}_{4}$ solution with $\mathrm{NaBH}_{4}$ followed by continues stirring for 30 minutes. In the beginning of process, a three varied AuNp samples prepared by the same recipe but different amount of PVA to study how the amount of stabilizers affect particle size and distribution of the synthesized gold nanoparticles. The amount of PVA added in appropriate quantities so that the final concentration of gold ${ }^{0}$ would be about $50 \%, 10 \%$ and $5 \%$ of PVA or less.

PVA is well known as a good stabilizer for metal nanoparticles based on research [11,12]. The reduction of $\mathrm{Au}^{3+}$ ion was visually confirmed as the reaction mixture turned red ruby within a few minutes. Particle size usually correspond with maximum wavelength $\left(\lambda_{\max }\right)$ observed in UV-Vis spectroscopy while full width half maxima (FWHM) related to size distribution. From the Table 1, it can be seen that $\lambda_{\max }$ decreased with the increasing amount of PVA while maximum wavelength $\left(\lambda_{\max }\right)$ usually correspond with particle size. This suggested that AuNp will be stabilized properly at concentration 5\% or less of PVA concentration. It is also clear that a lower PVA amount will result in broader SPR peak indicating a formation of nanoparticles with broader size distribution.

The amount of optimized PVA before will then be chosen to fabricate AuNp for further steps. As it was mentioned before that conjugating folic acid with AuNp's surface required two steps. The first step is to conjugate AuNp's surface with 4Atp as linker. In this conjugation, 4Atp was attached to AuNp's surface through it's sulfur atom. Several studies have successfully fabricated gold nanoparticles capped with thiolate molecules and indirectly confirmed the strong affinity of thiol groups against gold surface[13-15]. This conjugation was confirmed visually by the change in colour of the mixed solution from ruby red to deep purple blue as it was seen in Figure 1. SPR characteristic band of a gold nanoparticles linked with various molecules usually exhibit peak around 520-600 nm which is depend on particle size. Since interlinked molecules will also increase the size of a particle, a significance red shift on the SPR band will also be spotted if the attachment is success. Furthermore, an observation of SPR characteristic band change also be observed through the whole conjugation process. It was noticed that there was a significance different between AuNp 
before and after conjugation with FA and Rsv where the SPR band shifted from $522 \mathrm{~nm}$ for AuNp to $547 \mathrm{~nm}$ for FA-AuNp-Rsv conjugate. This result indicating an increase in particle size and also will be confirmed in the next investigation. Subsequently after the final conjugate was obtained, standard stability tests were performed using different medium and condition ( $\mathrm{pH} 7.4 ; 0.9 \% \mathrm{NaCl}$; bovine serum albumin; $1 \%$ cysteine). The final conjugate was stable in a long period of time (data not shown) and stored for next investigation.

\subsubsection{Fourrier transform infra red analysis}

The surface modification of AuNp has also been confirmed using FT-IR instruments. Pure PVA showed a characteristic bandwidth of $\mathrm{O}-\mathrm{H}$ stretching region at $3387 \mathrm{~cm}^{-1}$ while the absorption band was shifted at 3371 $\mathrm{cm}^{-1}$ for AuNp stabilized with PVA. This observed hydroxyl vibrational band in AuNp and the other shifted band confirmed the presence of PVA coating on AuNp's surface. 4Atp molecule posses a specific absorption band at $2548 \mathrm{~cm}^{-1}$ due to the presence of thiol $(\mathrm{S}-\mathrm{H})$ group which was disappeared after conjugation with AuNp's surface while the other absorption band were either shifted or disappear as well. This result confirmed the conjugation of $4 \mathrm{Atp}$ to AuNp has taken place via thiol group. Furthermore, as it was mentioned before that FA and AuNp conjugation take place by forming amida bond between amine group from 4Atp with carboxylic group from FA. Therefore it can be confirmed from the peak with several absorption bands at 1647 and $1678 \mathrm{~cm}^{-1}\left(\mathrm{C}=\mathrm{O}\right.$ amide bond) and also broad bands on ranged from 3700 to $3000 \mathrm{~cm}^{-1}(\mathrm{~N}-\mathrm{H}$ vibration of amide bond). This result confirms the formation of amide bond was occurred along with the conjugation of folic acid in AuNp's surface. On the final step as it can be observed in Figure 2, a characteristic band of pure Rsv at $3306 \mathrm{~cm}^{-1}$ (phenolic O-H groups stretch), $1384 \mathrm{~cm}^{-1}$ (C-O stretching), $1585 \mathrm{~cm}^{-1}$ (C-C olefinic stretching) and around $1600 \mathrm{~cm}^{-1}$ (C-C aromatic double bond stretching). Those specific absorption bands were remain present in the final conjugate with a slight shift indicating the formation of resveratrol conjugation in the final FA-AuNp-Rsv conjugate. These result also correspond with findings on other research [16].

\subsubsection{Size, distribution and zeta potential measurements}

Particle size of the AuNp, PVA stabilized AuNp and final conjugate FA-AuNp-Rsv was measured by dynamic light scattering (DLS) method and it was found out to be $1.8 \mathrm{~nm}, 76.0 \mathrm{~nm}$ and $86.9 \mathrm{~nm}$ respectively. These results was reasonable considering an increase in particle size is due to attachment of several molecules on the surface. In the beginng of the step, it was also noted that AuNp produced without the addition of PVA result in polydispersity index (PDI) value at 0.481 indicating a more polydispersed particles, while that of stabilized with PVA result in almost uniform size with PDI at 0.178 . This result was indicating that the use of PVA could lower the degree of polydispersity in the fabrication of gold nanoparticles.

Zeta potential of FA-AuNp-Rsv conjugate was found to be $-29.0 \mathrm{mV}$ and it serves as a good proof of nanoparticle's stability in aqueous medium. This value indicates that the conjugate were stable due to sufficient and acceptable electrostatic repulsion. These results were later also supported by stability testing of the conjugate in different media. Zeta potential is also one of the important value to determine the stability of metal nanoparticles. The surface charge around the nanoparticles could suspend the repulsion between particles and thus avoid aggregation, and so far zeta potential around $-30 \mathrm{mV}$ is considered suitable to maintain stability [17].

Table 1. Absorption, maximum wavelength and FWHM value of AuNp stabilized with different amount of PVA.

\begin{tabular}{ccccc}
\hline Formulation code & PVA (mg) & $\lambda_{\max }$ & (A) & FWHM \\
\hline F1 & 25 & 526.74 & 0.772 & 156.78 \\
F2 & 12.5 & 527.99 & 0.705 & 173.71 \\
F3 & 2.5 & 527.07 & 0.599 & 614.22 \\
F4 & 0 & 528.90 & 0.779 & 207.2 \\
\hline
\end{tabular}

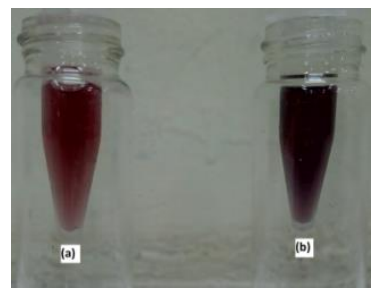

Figure 1. Colour changed from PVA stabilized AuNp (a) and 4Atp-AuNp conjugate (b). 


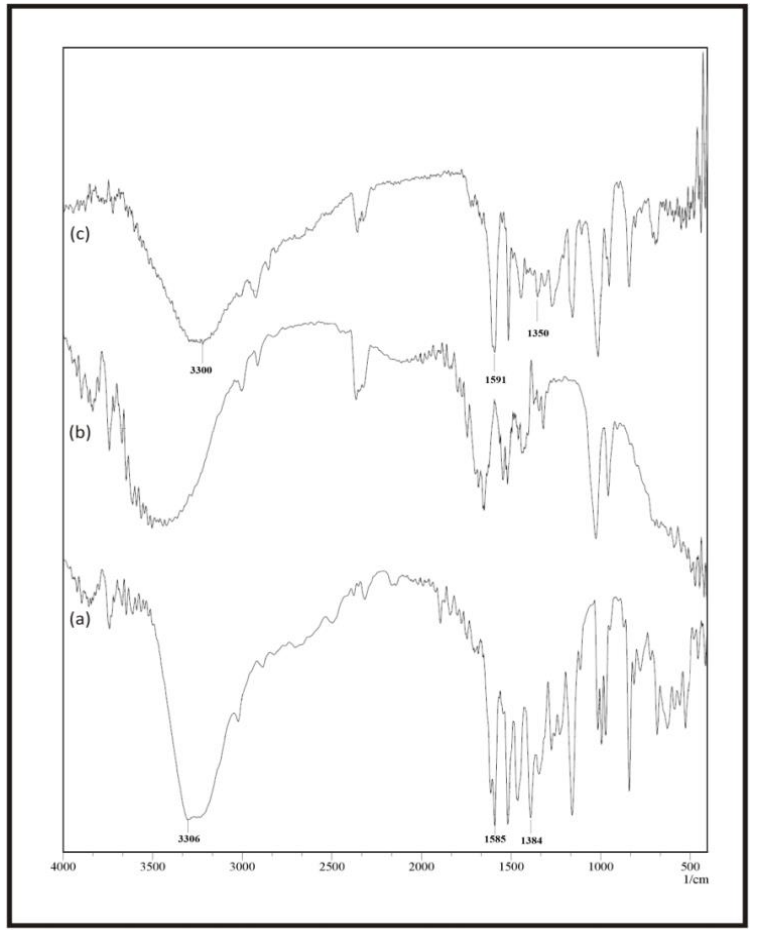

Figure 2. FT-IR spectra of (a) pure Rsv, (b) FA-AuNp conjugate and (c) FA-AuNp-Rsv conjugate.

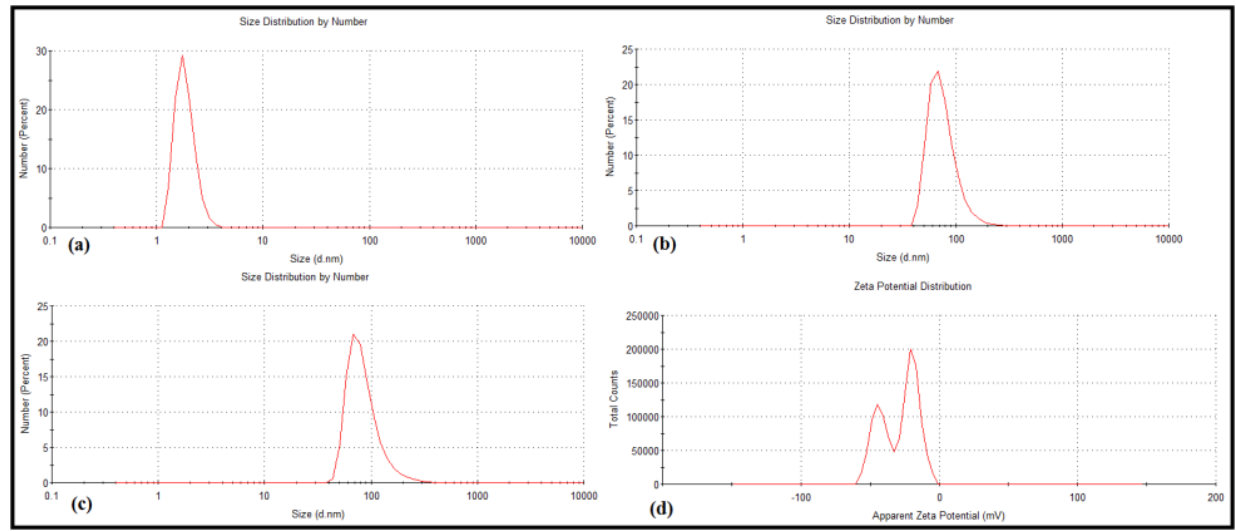

Figure 3. Particle size analysis of (a) AuNp, (b) PVA stabilized AuNp, (c) FA-AuNp-Rsv and (d) zeta potential distribution of FA-AuNp-Rsv.

\subsection{Entrapment efficiency}

The amount of Rsv in FA-AuNp-Rsv conjugate were measured indirectly by measuring the free Rsv present in the filtrate of nanoconjugate using HPLC method. Standard curve of free Rsv obtained were $\mathrm{y}=$ $120854 x+113973,(R=0,9987)$. Furthermore by measuring the sample triplicate an average concentration of $0.0397 \mathrm{mg} / \mathrm{ml}(39.78 \mathrm{ppm})$ were measured. Thus this result revealed that about $79.57 \pm 0.18 \%$ of resveratrol was conjugated to gold nanoparticles conjugate. This result is higher from other experiment by kumar et al in which about $20 \%$ of resveratrol was successfully conjugated with gold nanoparticles [17].

\subsection{Cytotoxicity Test}

In this research, we were trying to increase the effectiveness of resveratrol as an anti cancer agent against breast cancer by the use of AuNp with folate residues as a homing device. MCF-7 cell line has been used as a breast cancer model cell to study cytotoxic effect of various compounds. Joshi $e t$ al studied the enhancement of chloroquine conjugated to gold nanoparticles (GNP-Chl) against MCF-7 cell line to portray it's efficacy against breast cancers [13]. The presence of folate receptors on MCF-7 cell line makes it a perfect candidate in this study since our carrier use folic acid as an introducing agent. 
A

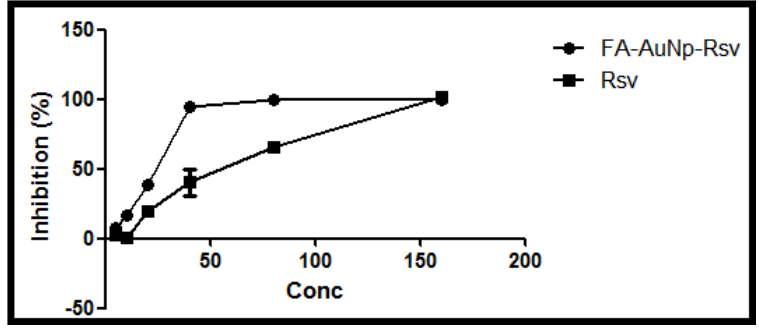

B

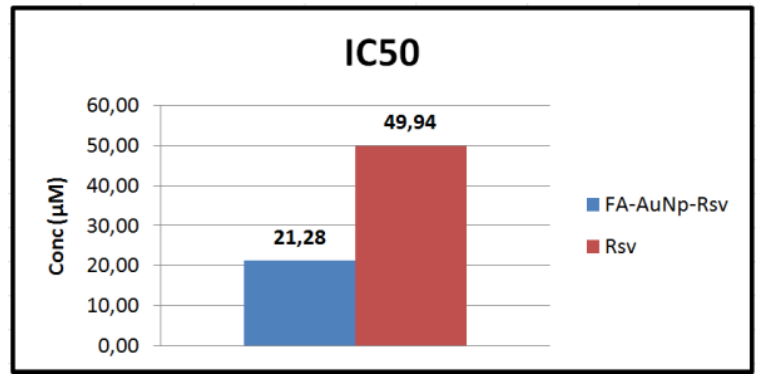

Figure 4. (a) Concentration vs \% inhibition curve of FA-AuNp-Rsv and Rsv, (b) $\mathrm{IC}_{50}$ comparison between FaAuNp-Rsv and Rsv.

In order to test the enhancement of resveratrol potency in conjugate, we compare the effect of various concentration FA-AuNp-Rsv conjugate and free Rsv on MCF-7 cell line using MTT assay. Cells were treated with various concentrations $(5,10,20,40,80$ and $160 \mu \mathrm{M})$ of resveratrol in FA-AuNp-Rsv conjugate and compared with that of bare resveratrol and incubated for 24 hours. The cells response were determined as a percent of inhibition calculated using the MTT assay. As it was shown in Figure 4(a), the inhibition of FAAuNp-Rsv conjugate by every dose was higher then that of free Rsv. The conjugate also gave inhibition nearly twice higher than bare resveratrol in every concentration. This result is reasonable and can be explained in two ways. The first is that metal nanoparticles has been proved to be possess a cytotoxic activity thus can act synergistically with anti-cancer agents $[18,19]$. Secondly folic acid on FA-AuNp-Rsv conjugate can act as a helper that increase the amount of conjugate presence on the MCF-7 cells surface through binding with folate receptors and by that maximize the drugs that enter the cells.

Furthermore, the IC50 value then calculated from linear curve of log concentration vs Normalized inhibition (\%) using Graphpad Prism 5 software. It was found that FA-AuNp-Rsv gave lower value (21.28 \pm $1.04 \mu \mathrm{M})$ than Rsv $(49.94 \pm 1.06 \mu \mathrm{M})$. Furthermore, two-way anova analysis showed that there were significant differences between each inhibition result for all concentration samples $(\mathrm{P}<0.05)$. There were also statistical differences between Fa-AuNp-Rsv and Rsv group $(\mathrm{P}<0.05)$. Thus we can conclude from our data that resveratrol's potency has been enhanced using gold nanoparticles bearing folate residues as a carrier. In earlier study by Kim et al, treatment of resveratrol gave an induced condensation and nuclear fragmentation on the observed MCF-7 cells and suggested that apoptosis induction as the main mechanism of inhibitory activity [20].

\section{CONLUSION}

Resveratrol conjugated to gold nanoparticles decorated with folate has been successfully synthesized and tested for their cytotoxicity activity in this study. The further characterization data showed that the conjugation was successful, well dispersed and stable. Also the potency of resveratrol in the final nanoconjugate was higher than that of resveratrol alone. It was concluded from this research that the conjugation resveratrol to gold nanoparticles decorated with folic acid could elevate the effectiveness of reseratrol as an alternative to cancer therapy. These results could be a valuable informations for another enhancement of low bioavailability compounds.

\section{MATERIALS AND METHODS}

\subsection{Materials}

Chloroauric acid $\left(\mathrm{HAuCl}_{4}\right)$ solution, sodium borohydride $\left(\mathrm{NaBH}_{4}\right)$, polyvinyl alcohol/PVA (Mw 67000), 4-Aminothiophenol (4ATP), 1-ethyl-3-(3-dimethylaminopropyl)carbodiimide hydrochloride (EDC), 
$\mathrm{N}$-hydroxysuccinimide (NHS), folic acid and resveratrol were purchased from Sigma-Aldrich, USA. Ethanol, deionised water, acetonitril, and all other reagents and solvents were of analytical grade.

\subsection{Synthesis of PVA stabilized AuNp}

In short a three different AuNp solution were prepared to study effect of stabilizers consentration. Briefly, a solution of $0.625 \mathrm{ml} \mathrm{HAuCl}_{4} 0.01 \mathrm{M}$ were dissolved and stirred in deionized water. Shortly after, an ice cold $0.1 \mathrm{ml}$ of freshly prepared $\mathrm{NaBH}_{4} 0.1 \mathrm{M}$ solution were added to the solution and the stir continued for 15 minutes. The colour rapidly changed from pale yellow to ruby red indicated a formation of gold nanoparticles. After that, a series of aqueous solution containing varied amount of PVA added to those three different AuNp solution. The PVA stabilized AuNp were obtained and kept for next studies.

\subsection{Synthesis of FA-AuNp Conjugate}

FA was conjugated with AuNp through 4Atp as linker. 4Atp functionalized AuNp was prepared according to the method present in literature [21]. In the first step, a varied ethanolic solution of 4Atp (0.3 M) that has been prepared before was added drop wise to the stirred solution of $\mathrm{AuNp}(10 \mathrm{ml})$. The solution then stirred for about an hour to complete the conjugation. The colour of the solution changed from ruby red to purple blue indicated that the attachment has taken place to form 4Atp-AuNp conjugate.

FA solution $(8.8 \mathrm{mg})$ was activated by addition of freshly prepared EDC $(1 \mathrm{ml}, 20 \mathrm{mM})$ and NHS $(1 \mathrm{ml}$, $50 \mathrm{mM}$ ) in DMSO. The solution was stir for up to 3 hours to complete the activation. The solution later added to the $4 \mathrm{Atp}-\mathrm{AuNp}$ conjugate to form FA-AuNp conjugate through 4Atp link. Stirring was continued for the next 2 hours to complete the reaction. An observation also conducted to characterize using spectrophotometer UV-Vis (Shimadzu) and FT-IR to confirm the attachment for each step.

\subsection{Synthesis of FA-AuNp-Rsv conjugate}

Resveratrol conjugation to AuNp procedure was done according to the method in literature with slight modification [17]. $1 \mathrm{ml}$ solution of resveratrol $(2 \mathrm{mM})$ in ethanol was added to FA-AuNp conjugate solution and kept for continues stirring for 2 hours in room temperature and the conjugate result (FA-AuNp-Rsv) was purified using a centrifuge tube filters (MWCO of 10kDa). The pellet obtained and washed several times and suspended in water for further verification.

\subsection{Characterization}

The absorption spectra of AuNp and conjugates are observed using UV-Vis spectroscopy (Shimadzu UV-1601). Sample solutions were put in $10 \mathrm{~mm}$ path length quartz cuvettes and measurement conducted in range of 200-800 $\mathrm{nm}$ wavelength for characteristic surface Plasmon resonance (SPR).

Fourrier transform infra red analysis was performed using Shimadzu FT-IR type 8400s. Samples were freeze dried and mixed with $\mathrm{KBr}$ powder and measured at $400-4000 \mathrm{~cm}^{-1}$ wavelength.

Particle size, distribution and zeta potential were measured on a particle size analyzer (Zetasizer Nano ZS, Malver Instruments, Ltd., UK). Samples were suspended in deionised water and placed in cell and measured at room temperature.

\subsection{Entrapment efficiency}

The concentration of resveratrol in FA-AuNp-Rsv conjugate were measured using high performance liquid chromatography instruments with UV-Vis detector based on methods presented literature with slight modifications [22]. The standard curve of resveratrol initially prepared using different concentration of resveratrol ranging from 1 to $50 \mu \mathrm{g} \mathrm{ml}^{-1}$ and an injection of $20 \mu \mathrm{l}$ was applied for all standards and samples. The chromatographic separation was conducted with Phenomex $\mathrm{C}_{18}$ column and detection was carried out at $306 \mathrm{~nm}$. The mobile phase was a mixture of methanol: $10 \mathrm{mM}$ potassium dihydrogen phosphate buffer $(\mathrm{pH}$ 6.8): acetnitrile (63:30:7) at a flow rate of $1 \mathrm{ml} / \mathrm{min}$.

Encapsulation efficiency (EE) was determined by measuring the concentration of free Rsv in filtrate obtained from purification of FA-AuNp-Rsv conjugate by ultracentrifugation. EE will then be measured triplicate and calculated as below:

$$
\mathrm{EE}(\%)=\frac{\text { Rsv initial concentration added }- \text { Rsv concentration on filtrate }}{\text { Rsv initial concentration added }} \times 100 \text { (Eq. 1) }
$$




\subsection{Cytotoxicity test}

MTT test was chosen as a method to determine the cytoxic activity in the final step of this research. Six varied concentration ranging from 5 to $160 \mu \mathrm{M}$ were prepared for both groups of FA-AuNp-Rsv solution and free Rsv solution. Concentration were calculated based on the concentration of resveratrol in both samples. The samples later tested against human breast adenocarcinoma cell line (MCF-7). Cells were grown using DMEM medium in 96-well plates ( 4500 cells in $100 \mu \mathrm{L}$ per well), suplemented with $10 \%$ fetal bovine serum and antibiotics at $37^{\circ} \mathrm{C}$ with $5 \% \mathrm{CO}_{2}$. Samples were added to each well in triplicate and after 24 hours of incubation, $10 \mu \mathrm{L}$ MTT reagent $(5 \mathrm{mg} / \mathrm{ml})$ was administered to each well and incubation was continued for the next 4 hours. Subsequently the purple formazan crystals were dissolved in ethanol and the absorbance was read at a wavelength of $595 \mathrm{~nm}$. The IC50 values were determined from the dose-response curves using GraphPad 5 Prism software. Statistical two-way variance analysis also conducted using SPSS 22.0 software.

\subsection{Statistical analysis}

Statistical analysis were performed for all data results in cytotoxicity test using one-way variance analysis with confidence level of 95\%, with SPSS 22.0 statistics software. All the experiments were performed triplicate.

Acknowledgements: This research financially supported by the Directorate of Research and Community Engagement (DRPM), Universitas Indonesia through PITTA 2018.

Author contributions: Concept - S.S., M.S.; Design - S.S., M.S.; Supervision - S.S. A.M.; Materials - S.S; Data Collection and/or Processing - M.S., S.S.; Analysis and/or Interpretation - A.M., S.S., M.S.; Literature Search - M.S., S.S.; Writing - M.S., S.S.; Critical Reviews - S.S., A.M., M.S.

Conflict of interest statement: The authors declared no conflict of interest.

\section{REFERENCES}

[1] Desantis CE, Jiemin, Sauer AG, Newman LA, Jemal A. Breast cancer statistics , 2017: racial disparity in mortality by state. CA Cancer J Clin. 2017; 67(6): 439-448. [CrossRef]

[2] Davidov-Pardo G, McClements DJ. Resveratrol encapsulation: designing delivery systems to overcome solubilitystability and bioavailability issues. Trends Food Sci Tech. 2014; 38(2): 88-103. [CrossRef]

[3] Murtaza G, Latif U, Najam-Ul-Haq M, Sajjad A, Karim S, Akhtar M, Hussain I. Resveratrol: an active natural compound in red wines for health. J Food Drug Anal. 2013; 21(1): 1-12. [CrossRef]

[4] Walle T, Hsieh F, DeLegge MH, Oatis jr JE, Walle UK. High absorption but very low bioavailability of oral resveratrol in humans. Drug Metab Dispos. 2004; 32(12): 1377-1382. [CrossRef]

[5] Joseph MM, Sreelekha TT. Gold nanoparticles-synthesis and application in cancer management. Recent Pat Mater Sci. 2014; 7: 8-25. [CrossRef]

[6] Brust M, Walker M, Bethell D, Schiffrin DJ, Whyman R. Synthesis of thiol-derivatised gold nanoparticles in two phase liquid-liquid system. J Chem Soc Chem Comm. 1994; 7: 801-802. [CrossRef]

[7] Alanazi FK, Radwan AA, Alsarra IA. Biopharmaceutical applications of nanogold. Saudi Pharm J. 2010; 18: $179-193$. [CrossRef]

[8] Dreher MR, Liu W, Michelich CR, Dewhirst MW, Yuan F, Chilkoti A. Tumor vascular permeability, accumulation, and penetration of macromolecular drug carriers. J Natl Cancer I. 2006; 98(5): 335-344. [CrossRef]

[9] Bertrand N, Wu J, Xu X, Kamaly N, Farokhzad OC. Cancer nanotechnology: The impact of passive and active targeting in the era of modern cancer biology. Adv Drug Deliver Rev. 2014; 66: 2-25 [CrossRef]

[10] Kumar SSD, Mahesh A, Antonira MG, Rathore HS, Houreld NN, Kandasamy R. Cellular imaging and folate receptor targeting delivery of gum kondagogu capped gold nanoparticles in cancer cells. Int J Biol Macromol. 2017; 109: 220230. [CrossRef]

[11] Khanna PK, Gokhale R, Subbarao VVVS, Vishwanath AK, Das BK, Satyanarayana CVV. PVA stabilized gold nanoparticles by use of unexplored albeit conventional reducing agent. Mater Chem Phys. 2005; 92: $229-233$. [CrossRef] 
[12] Eun Jung Kim, Jeong Hyun Yeum, Jin Hyun Choi. Effects of polymeric stabilizers on the synthesis of gold nanoparticles. J Mater Sci Technol. 2014; 30(2): 107-111 [CrossRef]

[13] Joshi P, Chakraborti S, Ramirez-Vick JE, Ansari ZA, Shanker V, Chakrabarti P, Singh SP. The anticancer activity of chloroquine-gold nanoparticles against MCF-7 breast cancer cells. Colloids Surf. B. 2012; 95: 195-200. [CrossRef]

[14] Zhang Z, Jia J, Lai Y, Ma Y, Weng J, Sun L. Conjugating folic acid to gold nanoparticles through glutathione for targeting and detecting cancer cells. Bioorg Med Chem. 2010; 18: 5528-5534. [CrossRef]

[15] Mansoori GA, Brandenburg KS, Shakeri-Zadeh A. A comparative study of two folate-conjugated gold nanoparticles for cancer nanotechnology applications. Cancers. 2010; 2(4): 1911-1928. [CrossRef]

[16] Tomoaia G, Horovitz O, Mocanu A, Nita A, Avram A, Racz CP, Soritau O, Cenariu M, Tomoaia-Cotisel M. Effects of doxorubicin mediated by gold nanoparticles and resveratrol in two human cervical tumor cell lines. Colloids Surf B. 2015; 135: 726-734. [CrossRef]

[17] Kumar CG, Poornachandra Y, Mamidyala SK. Green synthesis of bacterial gold nanoparticles conjugated to resveratrol as delivery vehicles. Colloids Surf B. 2014; 123: 311-317. [CrossRef]

[18] Loutfy SA, Al-Anshary N, Abdel-Ghani NT, Hamed AR, Mohamed MB, Craik JD, Eldin TA, Abdelah AM, Hussein Y, Hasanin MTM, Elbehairi SE. Anti-proliferative activities of metalic nanoparticles in an in vitro breast cancer model. Asian Pac J Cancer Prev. 2015; 16(14): 6039-6045. [CrossRef]

[19] Selim ME, Hendi AA. Gold nanoparticles induce apoptosis in MCF-7 human breast cancer cells. Asian Pac J Cancer Prev. 2012; 13(4): 1617-1620. [CrossRef]

[20] Kim YA, Choi BT, Lee YT, Park DI, Rhee SH, Park KY, Choi YH. Resveratrol inhibits cell proliferation and induces apoptosis of human breast carcinoma MCF-7 cells. Oncol Rep. 2004; 11: 441-446. [CrossRef]

[21] Patel UB, Metha VN, Kumar MA, Kailasa SK. 4-Aminothiophenol functionalized gold nanoparticles as colorimetric sensors for the detection of cobalt using UV-Visible spectrometry. Res Chem Intermed. 2013; 39(2): 771-779. [CrossRef]

[22] Singh G, Pai RS. A rapid reversed-phase HPLC method for analysis of trans-resveratrol in PLGA nanoparticulate formulation. ISRN Chromatogr. 2014; Article ID 248635. [CrossRef]

This is an open access article which is publicly available on our journal's website under Institutional Repository at http://dspace.marmara.edu.tr. 